Schmerz 2021 · 35:434

https://doi.org/10.1007/s00482-021-00583-0

Angenommen: 16. August 2021

Online publiziert: 2 . September 2021

๑) Springer Medizin Verlag GmbH, ein Teil von Springer Nature 2021

\section{Cannabis als Schmerzmedizin - zu viele Fakes, zu wenig Fakten}

Michael A. Überall

Privates Institut für Neurowissenschaften, Algesiologie und Pädiatrie - IFNAP, Nürnberg, Deutschland
Leserbrief zu

Maier C, Glaeske G (2021) Green Rush schlimmer als befürchtet? Schmerz 35:185-187. https://doi.org/10.1007/s00482-021-00560-7

Vielen Dank für diesen Beitrag, der mit seinen Ausführungen den Finger in eine Wunde der mittlerweile alltäglichen Versorgung von Menschen mit medizinischem Cannabis legt, die angesichts des medialen Hypes in Laien- und Fachmedien gerne klein geredet wird: die fragwürdige medizinische Sinnhaftigkeit und die weitestgehend fehlende wissenschaftliche Evidenz für die Verordnung von Cannabis als (Schmerz-)Medizin.

Angesichts der in ihren grundsätzlichen kritischen Ausführungen zu begrüßenden Stellungnahme fallen einige terminologische Fehler auf, die so nicht stehen gelassen werden sollten und einer Korrektur bedürfen.

So wird z. B. bereits im ersten Absatz des Artikels (Zeile 18) der Wirkstoff Dronabinol mit dem Wirkstoff Nabiximols (letzterer auch noch in der umgangssprachlich „eingedeutschten“ und um das abschließende "s" gekürzten Schreibweise) in missverständlicher Form „in einen Topf geworfen".

Ebenfalls auf der ersten Seite (zweite Spalte, Zeile 14) wird fälschlicherweise behauptet, dass Nabilon ein „Fertigarzneimittel mit Cannabidiol" sei.

Beide Fehler mögen auf den ersten Blick und angesichts des großen Ganzen sicherlich unbedeutend erscheinen, sind aber gerade bzgl. der grundlegenden Bedeutung des Themas und der zunehmenden werblichen Einflussnahme auf Berichterstattung, Anzeigen in Fach- und Laienmedien sowie medizinischen Kongressen unnötig und lassen den (sicherlich unbegründeten, in jedem Fall jedoch unnötigen) Verdacht auf eine mangelnde Sorgfalt der beiden Autoren auch bei anderen wichtigen (und richtigen) Aussagen dieses Beitrags aufkommen. Dies sollte angesichts der hohen Bedeutung dieses Themas vermieden werden.

Korrespondenzadresse

PD Dr. med. Michael A. Überall

Privates Institut für Neurowissenschaften, Algesiologie und Pädiatrie - IFNAP Nordostpark 51, 9411 Nürnberg, Deutschland Michael.Ueberall@ifnap.de

Interessenkonflikt. M.A. Überall gibt an: Finanzielle Interessen: Honorare für Vortragstätigkeiten und Beratertätigkeit: Allergan, Almirall, Amicus Therapeutics, Aristo Pharma, Bionorica, Esanum, GlaxoSmithKline, Grünenthal, HAPA Medical, Hexal, IMC, Kyowa-Kirin, Labatec, Mucos, Mundipharma, Nestle, Pfizer, Recordati, Servier, SGP-Pharma, Shionogi, Spectrum Therapeutics, Strathmann, Teva und Tilray. Geschäftsführer der O. Meany-MDPM GmbH Nürnberg. Nichtfinanzielle Interessen: Leiter des privaten Instituts für Neurowissenschaften, Algesiologie und Pädiatrie, Nürnberg. Präsident der Deutschen Schmerzliga (DSL) e. V., Vizepräsident der Deutschen Gesellschaft für Schmerzmedizin (DGS) e.V. 